\title{
Analysis and Explanation of Snooker's Sports Culture and Communication Mode
}

\author{
Yiting Fan ${ }^{1}$ \\ ${ }^{1}$ Xi’an Medical University, Xi’an, 710000
}

Keywords: Snooker movement; cultural connotation; mode of communication

\begin{abstract}
The snooker campaign has always been full of gentlemen's colors. As the times develop, it also has more cultural qualities. The snooker movement in our country has also gone through four stages from the initial stages of budding, stagnation, recovery and development to rapid development. The snooker movement has similarities with our country's traditional cultural etiquette, which can promote the construction of our national quality and the development of sports. And now, when the snooker sports culture spreads, it often faces problems of local cultural exclusion and cultural bottlenecks. Snooker culture needs to be guided through normal dissemination and innovation of Snooker's sports culture, so that snooker sports culture is popularized and developed in China and around the world.
\end{abstract}

\section{Introduction}

Although we do not know the specific origin of billiards, there are five or six hundred years of history. It is an ancient and connotative gentleman movement. The culture of the snooker movement can have a positive impact on China's traditional culture. It has developed rapidly in China and even in the whole world. Now everyone wants to play no matter who is young or old. You can bring the club to the next game, which shows the popularity of the snooker campaign.

\section{The Cultural Connotation of the Snooker Movement}

\subsection{Gentleman Cultural Connotation}

The gentleman culture is a kind of social culture that combines the aristocratic spirit and certain values of various social strata. Nowadays, gentlemen who are modest, well-dressed, well-dressed, and respectful of others are often called gentlemen. This also reflects that the society's longing for and pursuit of civilized friendship and harmony, and the snooker movement embodies this gentleman culture. Billiards are the first to be held within the aristocracy. Therefore, participants need to abide by strict etiquette and rules. They should be dressed neatly when playing, not excessively casual, swaying, and always paying attention to their actions to maintain their civilization. In formal competitions, shirts, vests, trousers, shoes, and bow ties are all essential. Although the times have changed dramatically and the snooker movement has become more diversified, the gentleman cultural connotation embodied in the snooker movement has not changed, which is also the charm of the snooker movement.

\subsection{Leisure Culture Connotation}

With the development of the snooker movement, not only the aristocrats and the urban poor have enjoyed the sport. Participants in the sport have been expanded into gentlemen, teachers, workers, or students by gentlemen and aristocrats. During the sports process, participants have become more active. What you get is relaxed and laid back. Now more people are pursuing spiritual enjoyment. Therefore, the recreational nature of the snooker movement is more in line with the needs of the society. It is a combination of movement and invigoration. It can both strengthen one's health and self-cultivation, fully enjoy the fun of playing, relax and release the mind. 


\section{Existing Problems in the Distribution of Snooker Sports Culture}

\subsection{Single Way of Propagation, Lack of Correct Leadership}

Since the birth of the snooker movement, the spread of the snooker movement has spread to foreign countries through carriers such as merchants and the military. This single culture has been used throughout the entire time to allow other people around the world to stay in the right place. The snooker movement has a shallow understanding of the culture of utensils and institutional culture, but little is known about the spiritual and cultural connotations of the snooker movement. However, the sports consciousness and sports concept are the core of sports culture. If we cannot understand this, we cannot really understand the true connotation of sports. And it is this single mode of transmission that hinders other people's perception of the sport culture of snooker.

\subsection{The Lack of Proper Guidance in the Distribution of Snooker Sports Culture}

In people's minds, the film or the actual billiard room is often accompanied by hooligans and fights. It is a place where people get mixed up. Therefore, the snooker movement is regarded as a kind of street rogue in people's subconscious. Culture, and teachers and parents will use it to educate students away from snooker sports venues. In the process of spreading the snooker's sports culture, colliding with the native culture will produce a new culture, namely the street rogue culture, and the original gentleman culture of the snooker movement will be replaced by oblivion. The snooker movement has spread all over the world. It not only has a single mode of transmission, but also lacks correct guidance for the spread of the snooker culture. Therefore, it is very difficult for the snooker sports culture to gain recognition from all parts of the world.

\section{The Correct Transmission Route of Snooker Sports Culture}

\subsection{Integrate Cultural Resources and Correctly Guide the Spread of Snooker Culture}

In today's multi-media era, the emerging media has given Snooker a chance to spread its culture. With multiple modes of communication, we must pay attention to correctly guiding the sports culture of the snooker so that the general public can fully and correctly understand the sports culture of Snooker. On the one hand, we must maximize the spread of the snooker culture. The spread of the culture of the snooker movement requires not only the use of television and the Internet, but also the full use of newspapers, magazines, and other print media, so that not only developed regions but also other regions can learn more about snooker sports culture. The deep spirit of Snooker's sports culture, its utensil culture and institutional culture all require people to have a deep understanding. If conditions allow, snooker competitions can be held in second-tier cities or second-tier cities to expand the spread and impact of snooker sports culture. On the other hand, we should correctly guide the spread of the culture of snooker culture. The rigors and behaviors of the participating athletes are strictly required to maintain the surrounding environment of the Snooker Movement, and to prevent gambling, fighting and fighting, to allow participants to have a clean entertainment environment. At the same time, for the snooker competitions, it is necessary to formulate regulations for related events. Both players and spectators must abide by them, reflecting the gentlemanly manner.

\subsection{Building a Multicultural Identity System}

Globalization has brought closer relations between various countries and ethnic groups. At the same time, it has also made contact with different cultural systems in various countries more frequent. Each ethnic group, when developing local culture, will inevitably communicate and interact with foreign cultures. The more diverse value of culture is an important feature to gain national sports cultural identity. When snooker culture spreads, it often collides with local culture, and snooker culture is often at a disadvantage and strongly rejected. Even if China's attention to the snooker movement has increased due to the influence of Ding Junhui, it cannot be said that we have fully accepted this culture. Therefore, in order for the snooker culture to spread better, it is necessary to abandon "cultural conservatism", to be more inclusive and understandable for the 
snooker culture, not to accept blindly and identify, and to accept the characteristics of the snooker movement itself. Based on the recognition of the differences, the harmonious development of the snooker sports culture and the native sports culture is realized.

\subsection{Innovative Snooker Sports Culture}

Diversified sports culture can be more welcomed and accepted by people. Therefore, the snooker movement must first advance with the times and enhance its attractiveness. This requires the Snookian sports culture to innovate, that is, from Snow. The game system was innovative. As snookers played too long and there were fewer competitions, many players and fans had fatigue and visual fatigue, which greatly reduced the recognition value of the sports culture of snooker. It is also the problem of commercialization of snooker. Therefore, you can shorten the game time by reducing the number of red balls or the game. On the other hand, it is necessary to innovate the cultural spirit of snooker. The snooker movement is a kind of gentleman culture. However, in order to realize its cultural diversity, it is not only necessary to rely on its gentlemen's culture, but also to meet the popular culture, in order to gain more recognition and better development. Fancy snooker games the combination of snooker players' superb skills and wonderful interpretations is an effective way to make snooker more diverse.

\section{Conclusion}

The snooker movement can promote the communication and communication of sports culture in the world. It embodies a strong gentleman culture and is conducive to the spread of moral etiquette. Therefore, it is necessary to correctly guide the spread of the snooker's sports culture, make the snooker movement more diversified, and innovate in order to gain more cultural approval.

\section{References}

[1] Du Xuefeng. Snooker Sports Culture Identity and Communication[J]. Journal of Sports Culture, 2011, (1): 153-155.

[2] Zhang Wei. Research on the Cultural Value of Developing Snooker Movement in China[J]. Journal of Qiqihar Teachers College,2009, (6):56-57.

[3] Zong Yanwei. Research on the Development of Professional Snooker Events in China[J]. Journal of Sports Culture, 2013, (3): 72-75. 